\title{
On bedload measurement performances of high- resolution acoustic (ACVP) and conductivity (CCP) profilers
}

\author{
Guillaume Fromant ${ }^{1, *}$, Thibaut Revil-Baudard ${ }^{1}$, Ryan S. Mieras ${ }^{2}$, David Hurther ${ }^{1}$, \\ Julien Chauchat ${ }^{1}$, Jack A. Puleo ${ }^{2}$ \\ ${ }^{1}$ Laboratory of Geophysical and Industrial Flow, 38041 Grenoble Cedex 9, France \\ ${ }^{2}$ Centre for Applied Coastal Research, University of Delaware, Newark, DE, USA
}

\begin{abstract}
Intense sediment transport experiments were performed in a gravity driven open-channel flow with two sizes of non-spherical acrylic particles of diameter $d_{p}=1.0 \mathrm{~mm}$ and $d_{p}=3.0 \mathrm{~mm}$, and a maximum packing volumetric concentration of $0.55 \mathrm{~m}^{3} / \mathrm{m}^{3}$. Two flow conditions were adapted to ensure the same sediment transport regime for particle sizes as sheet flows (Shields numbers above unity) with an equi-repartition of the total net sediment transport rate between the suspended load and the bedload (Suspension number around unity). An acoustic scattering-based system, the Acoustic Concentration and Velocity Profiler (ACVP) and electric conductivity probes, the Conductivity Concentration Profiler (CCP) with two different vertical resolutions of $1 \mathrm{~mm}\left(C C P_{1 \mathrm{~mm}}\right)$ and $2 \mathrm{~mm}\left(C C P_{2 \mathrm{~mm}}\right)$, were used to measure time-resolved and averaged concentration profiles across the bed-load and suspension layers. A detailed comparative analysis of concentration and sheet flow layer thickness measurements obtained with the two systems across both the suspension and the bedload layers is presented. The capabilities and limitations of the two flow measurement technologies are outlined. Average sediment concentration profiles were overestimated by $10 \%$ with the ACVP in the dense sheet layer when $\langle\phi(z)\rangle \gtrsim 0.35$, and by $100 \%$ with the CCP in the more diluted region when $\langle\phi(z)\rangle \lesssim 0.015$ and $\langle\phi(z)\rangle \lesssim 0.20$ for $C C P_{1 \mathrm{~mm}}$ and $C C P_{2 \mathrm{~mm}}$, respectively. Good agreement is found elsewhere between the three systems in terms of average and time-resolved concentration as well as bed level position and sheet flow layer thickness.
\end{abstract}

\section{Introduction}

Over the past two decades, considerable research efforts have been dedicated to the understanding and modeling of sediment transport processes under energetic flow forcing conditions for which bedload moves as a sheet flow. In this context, it is widely accepted that the geophysical community suffers from the lack of high-resolution flow measurement tools adapted to the study of sediment transport physics in energetic environmental flows [1], [2]. Indeed, the ability to profile sediment transport across the entire bottom boundary layer over

\footnotetext{
* Corresponding author: guillaume.fromant@,univ-grenoble-alpes.fr
} 
a mobile sediment bed is currently limited to a few measurement technologies. The limited measurement capabilities have limited advances in process-based modeling of sediment transport in contrast to empirical models commonly implemented in numerical engineering codes that are applied for long-term predictions of morphological evolution. Such predictions are known to be subject to large uncertainties and errors which is a main research concern in the context of adaptation to abrupt climate changes.

The high particle concentration range $(0.08<\phi<0.53$; where $\phi$ is volumetric concentration) and the limited vertical thickness (scaling with the particle size) of the sheet flow layer makes it particularly difficult to accurately measure the particle concentrations with conventional non-intrusive optical and acoustic flow measuring tools. The difficulty is mainly because light and sound propagation are subject to strong scattering-dominated attenuation. Novel techniques were developed, aiming at measuring local concentration and streamwise velocity measurements (eq. [6]-[8]). Only recently have advanced acoustic and conductivity techniques been developed [9], [10] and used to profile such complex particle flows at sufficiently high temporal $(O(0.1 \mathrm{~s}))$ and spatial $(O(0.001 \mathrm{~m}))$ rates to resolve the small turbulent flow scales involved in the benthic boundary layer sediment transport processes.

This paper reports on detailed measurements of particle concentration and velocity profiles under gravity-driven sheet flow currents involving two different measurement systems: an Acoustic Concentration and Velocity Profiler (ACVP) [9] and Conductivity Concentration Profilers (CCP) [10] with two vertical resolutions (1 mm versus $2 \mathrm{~mm}$ ). The main objective of this study is to evaluate the performances and limitations of the above-mentioned measurement techniques, the ACVP and the CCPs, in measuring time-resolved and average sediment concentration profiles over the entire sediment transport layer. Section 2 presents the experimental setup and protocol, Section 3 describes the measurement principles and calibration procedures of the ACVP. The results of the comparative analysis are discussed in Section 4 .

\section{Experiment}

\subsection{Experimental facility and flow conditions}

The experiments were carried out at the Laboratory of Geophysical and Industrial Flows (LEGI) in the LEGI/ENSE3 tilting flume (Figure 1). The experimental facility is extensively described in [5], [11]. The flume is $L=10 \mathrm{~m}$ long and $W=0.35 \mathrm{~m}$ wide, with an adjustable bed slope $S_{0}$. A particle pit that is $L_{p}=3 \mathrm{~m}$ long by $z_{p}=0.11 \mathrm{~m}$ deep is mounted at the channel bed $2 \mathrm{~m}$ upstream of the channel outlet (Figure 1). Irregularly shaped Poly-Methyl MethAcrylate (PMMA) particles were used with a density of $\rho_{p}=1,192 \mathrm{~kg} / \mathrm{m}^{3}$. Two particle sizes were used in separate experiments, the coarse S3 and fine S1 particles with median particle diameters, $d_{p}$, of 3.0 and $1.0 \mathrm{~mm}$, respectively. A specific flow condition (i.e., water discharge and bed slope) was set for each particle size to achieve similar Shields numbers. The bed slope was set to $0.0025 \mathrm{~m} / \mathrm{m}$ for S3 and to 0.0050 for S1, with an average water discharge flow rate, $Q_{f}$, of $0.028 \mathrm{~m}^{3} / \mathrm{s}$ and $0.021 \mathrm{~m}^{3} / \mathrm{s}$ for $\mathrm{S} 3$ and $\mathrm{S} 1$, respectively (Table 1). 


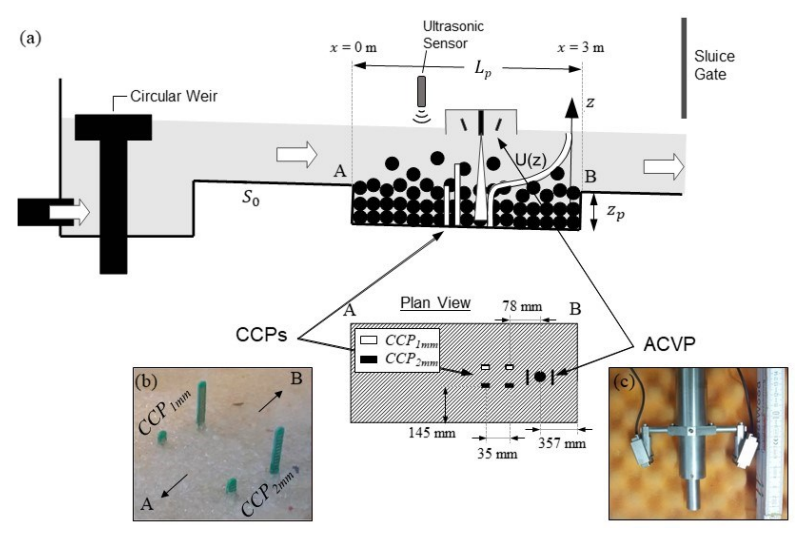

Fig 1: (a) Sketch of the experiment under active flow and location of the instruments. A and B delineate the upstream and downstream edges of the sediment pit, at $x=0 \mathrm{~m}$ and $x=3 \mathrm{~m}$, respectively. (b) Picture of the CCP probes deployed before an S3 run. (c) Picture of the ACVP.

Table 1: Flow and particles parameters for $\mathrm{S} 1$ and $\mathrm{S} 3$ experiments: bed slope $\left(S_{0}\right)$, run number $(N)$, fall velocity $\left(w_{s}\right)$, friction velocity $\left(u_{*}\right)$, water level $\left(H_{f}\right)$, flow rate $(Q)$, mean bulk flow velocity $(U)$, solid load per unit width $\left(q_{s}\right)$, Shields number $(\theta)$, suspension number $\left(w_{s} / u_{*}\right)$, dimensionless flux $(\psi)$, Reynolds number $(R e)$.

\begin{tabular}{ccccccccccccc}
\hline & $S_{0}$ & $w_{s}$ & $N$ & $u_{*}$ & $H_{f}$ & $Q$ & $U$ & $q_{s}$ & $\theta$ & $w_{s} / u_{*}$ & $\psi$ & $R e$ \\
\hline & $(-)$ & $(\mathrm{cm} / \mathrm{s})$ & $(-)$ & $(\mathrm{cm} / \mathrm{s})$ & $(\mathrm{m})$ & $\left(\mathrm{m}^{3} / \mathrm{s}\right)$ & $(\mathrm{m} / \mathrm{s})$ & $(\mathrm{m} / \mathrm{s})$ & $(-)$ & $(-)$ & $(-)$ & $(-)$ \\
\hline$S 3$ & 0.005 & 5.5 & 6 & 4.1 & 0.14 & 28 & 0.57 & $2.7 \times 10^{-4}$ & 1.2 & 1.3 & 3.7 & $8 \times 10^{4}$ \\
\hline$S 1$ & 0.0025 & 2.0 & 3 & 2.1 & 0.13 & 21 & 0.46 & $8.4 \times 10^{-4}$ & 1.7 & 1 & 6.3 & $6 \times 10^{4}$ \\
\hline
\end{tabular}

\subsection{Instrumentation setup}

The ACVP was positioned at the downstream end of the particle pit $(x=2.64 \mathrm{~m})$ to collect non-intrusive profiles of velocity and sediment concentration along the flow normal $z$ direction over a distance of $0.15 \mathrm{~m}$ above the initial flat bed level (Figure 1). Two pairs of CCPs were installed to measure concentration profiles along the flow normal $z$-direction. The vertical resolution was $1 \mathrm{~mm}$ for the pair of $C C P_{1 \mathrm{~mm}}$ probes, and $2 \mathrm{~mm}$ for the pair of $C C P_{2 m m}$ probes. Each pair of CCPs was aligned in the streamwise direction, $0.145 \mathrm{~m}$ away from the flume walls, and $0.035 \mathrm{~m}$ apart from each other in the streamwise direction (Figure 1b). The two CCPs with the same resolution were mounted with a vertical offset, overlapping by a few millimeters to increase the total vertical profiling range. The downstream probes of each CCP pair were positioned $0.078 \mathrm{~m}$ upstream from the ACVP at $x=2.56 \mathrm{~m}$. The streamwise shift avoided acoustic echoes from the CCP probes in the ACVP signal. An ultrasonic limnimeter was mounted on the trolley to measure the instantaneous water surface. The signal of the ultrasonic limnimeter was simultaneously recorded by the ACVP and the CCP systems allowing time referencing of all collected data.

\subsection{Experiment protocol}

The experimental protocol described in [11] was applied for each experimental run. Before the start of each run, the particle pit was filled with sediments, manually compacted and leveled as a flat uniform sediment bed of constant slope. In order to minimize the duration of transient flow until the target discharge was reached, the flume was filled with no particle movement until the still water level was slightly above the aimed normal flow depth. At this stage, the sluice gate at the flume outlet was still closed and a circular weir located at the 
upstream end of the flume bypassed any additional water discharge, resulting in a negligibly low flow rate with no sediment entrainment over the entire test section. An initial topographic survey of the particle pit was carried out by towing the ACVP mounted on a trolley, along the flume centerline between positions B and A (Fig. 1). Survey data were used to estimate the initial sediment volume assuming a spanwise-uniform flat bed with a constant packed bed porosity of $45 \%$. The ACVP was positioned at $x=2.64 \mathrm{~m}$ (Figure 1 ) before starting the experiment. An experimental run was initiated by rapidly opening the sluice gate at the outlet of the channel, provoking a sudden water level drop that deactivated the circular overflowweir and transferred the entire flow discharge into the flume. The start time (i.e., $t=0 \mathrm{~s}$ ) was defined for each run as the instant the water level - recorded by the ultrasonic sensor dropped to $95 \%$ of its original level. After a period of transient flow of about $30 \mathrm{~s}$, the fluid flow and the particle transport layer became fully developed with a normal flow depth of $H_{f}$ $=0.14 \mathrm{~m}(0.13 \mathrm{~m})$ and a mean bulk flow velocity of $U=0.57 \mathrm{~m} / \mathrm{s}(0.46 \mathrm{~m} / \mathrm{s})$ for $\mathrm{S} 3(\mathrm{~S} 1)$. After a duration of about two minutes, the sluice gate was closed to end the run and the original still water level was recovered. A post-experiment topographic survey was conducted by traversing the ACVP along the flume from B to A (see Figure 1a). The topographic surveys are used to estimate the total transported volume of sediments for each run assuming spanwise topographic uniformity. The experimental protocol was repeated for $N=6$ runs for the coarse particles (S3) and $N=3$ runs for the fine particles (S1) which provided a sufficient amount of data to minimize the bias errors of the statistical flow quantities. For each run, $z=0 \mathrm{~m}$ was defined as the initial "no flow" bed elevation at $t=0 \mathrm{~s}$.

\section{High-resolution measurement systems}

\subsection{Conductivity and concentration profiler}

The CCP measures electrical conductivity of a fluid-particle mixture, which is related to volumetric particle concentration via Archie's Law [10], [12]. Each CCP probe has 32 plate electrodes spaced at either 1 or $2 \mathrm{~mm}$ vertical increments, resulting in a vertical profile of volumetric particle concentration, $\phi(z)$, spanning either 29 or $58 \mathrm{~mm}$, respectively. All of the CCPs recorded profiles at $8 \mathrm{~Hz}$. Each CCP was calibrated using the two-point calibration method outlined by [10]. The bed interface, $z_{b}(t)$, was determined following [10]. At concentrations greater than $0.08 \mathrm{~m}^{3} / \mathrm{m}^{3}$, the average distance between spherical particles is less than one particle diameter [13], and particle-particle interactions become important.

\subsection{Acoustic Concentration and Velocity Profiler}

The ACVP technology proposed by [9] provides time-resolved 1D vertical profiling of the two components velocity field (as $u$ and $w$ for streamwise and flow normal velocity components, respectively) and the volumetric sediment concentration. The latter is obtained from the inversion of acoustic intensity profile observations which is combined to the simultaneous and co-located velocity measurement to provide multi-directional particle flux profiles at spatio-temporal rates resolving a wide range of the turbulent flow scales. The velocity measurement principle relies on the use of multi-bistatic pulse-coherent Doppler technology [9]. The ACVP is composed of one central transmitter with two bistatic receivers in the streamwise plane. The ACVP was set to operate at an acoustic frequency of $1 \mathrm{MHz}$, with a pulse duration of $2 \mu \mathrm{s}$, allowing a vertical resolution of $1.5 \mathrm{~mm}$ and temporal resolutions of 78 and $4.9 \mathrm{~Hz}$ for the velocity and concentration measurements, respectively. The lower temporal resolution for the concentration measurements follows the validity of 
incoherent scattering conditions implying a mean particle cloud displacement between two consecutive pings (or echoes) of at least $1 / 4$ of the acoustic wavelength [14].

\subsection{Validation of acoustic transport rate measurements}

Before and after each run, topographic surveys were used to estimate the total transported particle volume following the towing procedure described in 2.3. The ACVP recorded acoustic intensity and velocity profiles while it was towed over the particle pit. The bed level based on topography bed scans, $z_{b}(x)^{\text {topo }}$, was defined by the peak value in the acoustic intensity profiles (Figure $2 \mathrm{~b}, 2 \mathrm{~d}$ ), at which the trolley speed can be estimated. The instantaneous velocities of the towed ACVP trolley relative to the fixed tilted flume were integrated over time to determine the instantaneous horizontal position of the trolley over the particle pit. Finally, the total transported particle volume, $V_{\text {Topo }}$, was determined by subtracting the topographic elevations before and after each run and integrated over the particle pit.

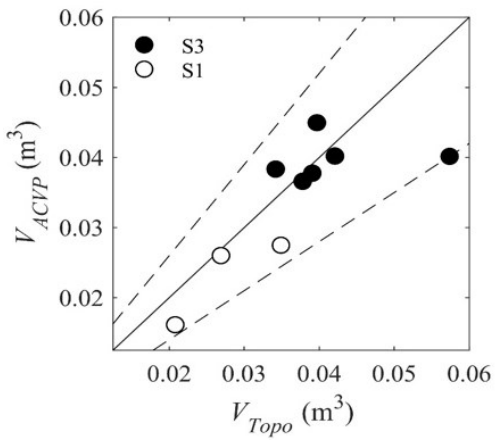

Fig. 2: Comparison between total transported volumes for all runs during S3 and S1 conditions, from the topographic $\left(V_{\text {Topo }}\right)$ and ACVP measurements $\left(V_{A C V P}\right)$. The solid line represents a 1:1 match; dashed lines correspond to $\pm 30 \%$ uncertainty
The acoustic data collected for each run were inverted following the methodology described in in [9], [11], yielding volumetric concentration profiles. The temporal evolution of the transported volume of particles at the location of the ACVP during each run was computed for S3 and S1 by cumulatively integrating the verticallyintegrated streamwise flux over time (Figure $3 a-3 b$ ),

$$
V_{A C V P}(t)=W \int_{0}^{t} \int_{z_{b}\left(t^{*}\right)}^{H_{f}} u\left(z, t^{*}\right) \phi\left(z, t^{*}\right) d z d t^{*}
$$

where $W$ is the channel width. The accuracy of the ACVP-derived particle fluxes was evaluated by comparing the total volume of particles transported for each run estimated from the ACVP measurements, with the total transported particle volume estimated from topographic surveys $V_{\text {Topo }}$ (Fig. 2). It can be seen that all acoustically estimated volumes are contained within an uncertainty range of $30 \%$ (Fig. 2; dotted lines) which strongly supports the transport rate measurement ability of the ACVP technology.

\section{Results}

\subsection{Time-resolved velocity and concentration measurements}

Fig. 3 presents time-space color plots of the time-resolved velocity profiles (ACVP) and concentration profiles (ACVP and CCPs) together with the time series of the undisturbed bed level (ACVP and CCPs), for a representative run of the S3 and S1 experiments. Only one run per condition is shown here for brevity. The initial phase of the experiments was characterized by a transient phase lasting about $40 \mathrm{~s}(60 \mathrm{~s})$ for $\mathrm{S} 3(\mathrm{~S} 1)$, with rapidly increasing velocities, reaching $1.1 \mathrm{~m} / \mathrm{s}(0.95 \mathrm{~m} / \mathrm{s})$ at $t=20 \mathrm{~s}(10 \mathrm{~s})$ for S3 (S1), before decreasing with time until $t=30 \mathrm{~s}(50 \mathrm{~s})$ for the S3 (S1) condition (Figure 3a, 3b). Concurrently, the color 
plots of concentration in Fig. 3 reveal that during the first $20 \mathrm{~s}$ of the experiments, the suspension steadily grew in time, reaching higher elevations.
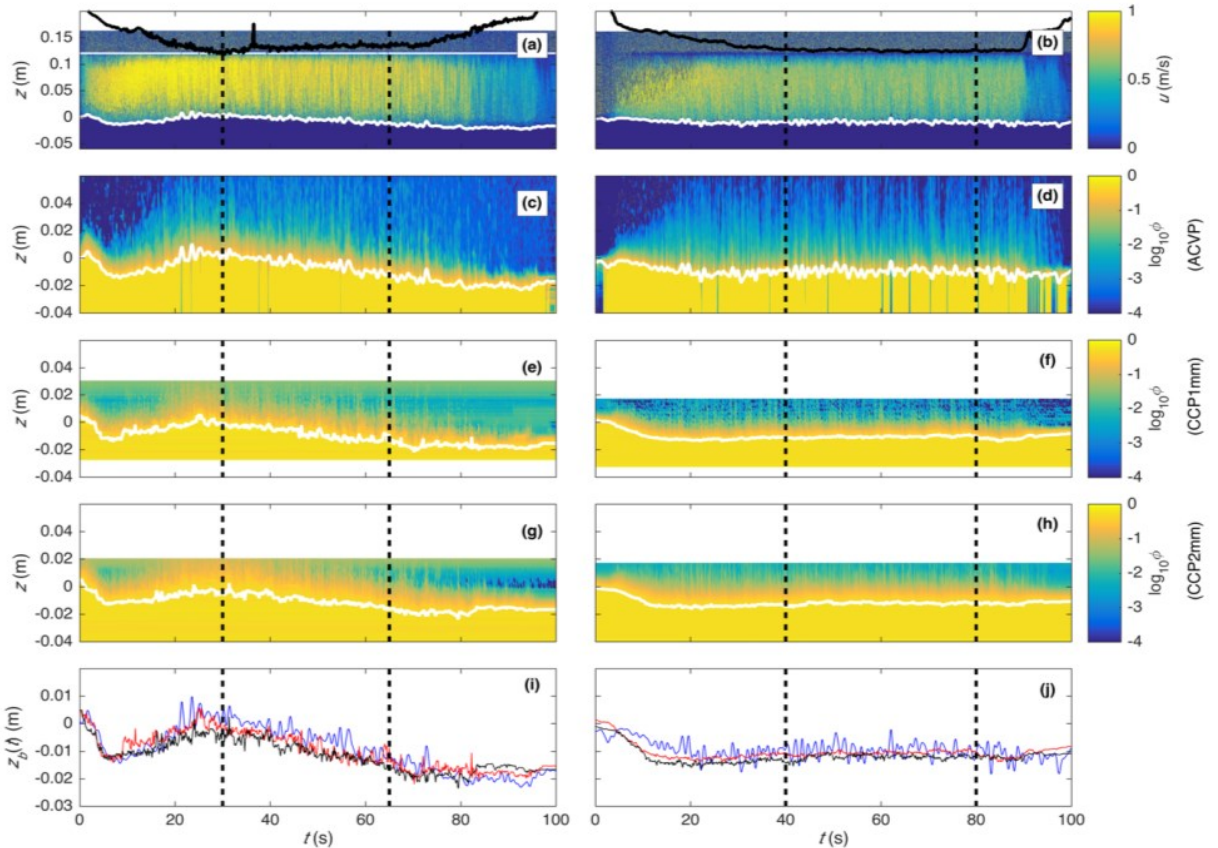

Fig. 3: Time-space plots of (a, b) streamwise ACVP velocity, (c, d) ACVP-derived concentration, (e, f) $C C P_{1 \mathrm{~mm}}$-derived concentration, $(\mathrm{g}, \mathrm{h}) C C P_{2 m m}$-derived concentration; and (i, j) bed interfaces derived from ACVP (blue line), $C C P_{1 \mathrm{~mm}}$ (red line) and $C C P_{2 \mathrm{~mm}}$ (black line) bed interfaces for S3 (left panel) and S1 (right panel) experiments. The black lines in (a,b) represent the instantaneous freesurface. The white lines are the bed interfaces estimated from ACVP and CCP data. The vertical dashed black lines denote the quasi-steady time interval over which the data are time-averaged.

After the transient phase, the flow reached a quasi-steady bed erosion phase, where the measured velocities stabilized over a period of time between $t=30-70 \mathrm{~s}$ for S3 and $t=$ $50-80 \mathrm{~s}$ for S1 (Fig. 3; vertical dashed black lines). This effect can also be visually inferred from the bed level evolution being horizontal for both the ACVP and CCPs over the same time windows (Fig. 3i, 3j), as well as from the steadiness of the free-surface elevation (black solid lines in the time intervals delimited by the two vertical dashed lines in Fig. 3a, 3b). At the end of each experiment, the sluice gate was closed, which led to a rapid decrease of the flow rate as the water level rose back to the initial still water level.

\subsection{Averaged flow quantities}

In order to increase statistical convergence and minimize statistical bias errors of measured mean flow quantities, the velocity, concentration and particle volume flux profiles were timeaveraged $(-$ ) over a portion of the quasi-steady time interval $(t \in[30 \mathrm{~s}, 65 \mathrm{~s}]$ and $t \in[40 \mathrm{~s}$, $80 \mathrm{~s}$ ] for the S3 and S1 experiments respectively - Fig. 3). Ensemble-averaging $(\langle\rangle)$ was then carried out over the $N$ repeated runs. The time-averaged velocity profiles in Figures $4 \mathrm{a}, \mathrm{b}$ reveal the existence of an inflection point (so-called "S-shape") typical under sheet flow conditions [5], [11]. The inflection point elevations also correspond well with the $8 \%$ volumetric concentration elevation (Fig. 4b,e), which separates the sheet flow layer $\langle\bar{\phi}\rangle>$ 

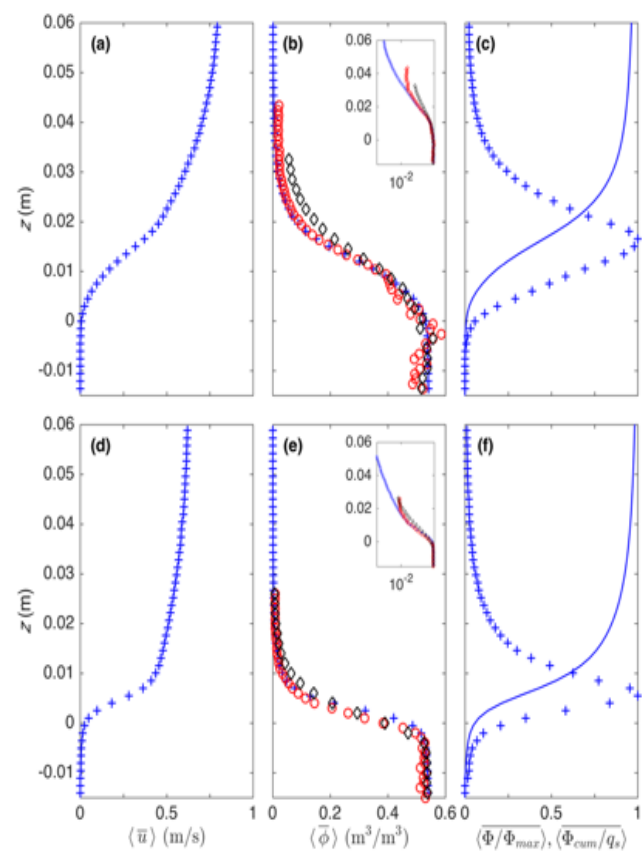

Fig. 4: Ensemble-time-averaged (a, d) velocity profiles, (b, e) concentration profiles, and (c, f) particle volume flux profiles for $\mathrm{S} 3(\mathrm{a}-\mathrm{c}$, top row) and $\mathrm{S} 1(\mathrm{~d}-\mathrm{f}$, bottom row). In panels $(\mathrm{b}, \mathrm{e})$, the concentration was measured by the ACVP ( + , plus), $C C P_{1 \mathrm{~mm}}$ (o, circles) and $C C P_{2 \mathrm{~mm}}$ (diamonds). The same concentration data are plotted in semilogarithmic scale (insets) to highlight the suspension layer (i.e., $\langle\bar{\phi}\rangle<0.08$ ). The blue lines in panels (c, f) are normalized, cumulatively integrated volume flux.
0.08 from the upper dilute suspension layer $\langle\bar{\phi}\rangle<0.08$. The shapes of the concentration profiles (Fig. 4b,e) are also similar to previously observed profiles for sheet flow conditions [15]. In the sheet flow layer, the concentration decreases almost linearly with $z$, whereas in the upper dilute region, an exponential decay typical of the suspension layer is observed (also called Rouse profile). For the sake of comparison, the root-mean-square (RMS) of the relative average concentration differences along the profile between the ACVP and CCPs was computed. Good agreement was observed over the entire profile, particularly in the range 0.02 $\left\langle\langle\bar{\phi}\rangle<0.35\right.$ for $C C P_{1 \mathrm{~mm}}$ (S3: RMS = $\left.0.018 \mathrm{~m}^{3} / \mathrm{m}^{3} ; \mathrm{S} 1: \mathrm{RMS}=0.014 \mathrm{~m}^{3} / \mathrm{m}^{3}\right)$. In the dense region $(\langle\bar{\phi}\rangle>0.35)$, the ACVP measures slightly larger mean concentration values than the CCPs, especially for the $\mathrm{S} 1$ condition (RMS = $0.042 \mathrm{~m}^{3} / \mathrm{m}^{3}$ for $C C P_{1 \mathrm{~mm}}$ and $\mathrm{RMS}=$ $0.031 \mathrm{~m}^{3} / \mathrm{m}^{3}$ for $C C P_{2 m m}$ ), while in the dilute region, the CCPs return higher concentration values. The over-prediction of concentration in the dilute region by $C C P_{2 m m}$ appears closer to the bed $(\langle\bar{\phi}\rangle \lesssim$ $0.2)$ compared to $C C P_{1 m m}(\langle\bar{\phi}\rangle \lesssim 0.02)$ and is probably related to the larger electrode spacing on the probe. Fig. $6 \mathrm{c}, \mathrm{f}$ show the time-averaged particle volume flux (blue + ) and cumulatively integrated timeaveraged volume flux profiles (blue line), which were directly estimated from the simultaneous and co-located velocity and concentration measurements provided by the ACVP. The peak of particle volume flux is located close to the transition between the sheet flow and the suspension layer $\phi \approx 0.08$, which is consistent with previous studies [11]. It reveals that there is a fairly equal partition between the bedload and suspended load contributions to the total net sediment transport rate. This is in good agreement with the corresponding Suspension number values around unity for both experiments.

\section{Conclusions}

A detailed comparative analysis of acoustic and conductivity-based measurements was carried out in gravity-current driven sheet flows with two sizes of light-weight acrylic particles with diameters of $1.0(\mathrm{~S} 1)$ and $3.0 \mathrm{~mm}(\mathrm{~S} 3)$. The measurement performances and limitations of the systems are evaluated for the vertical profiling across the sheet flow and suspension layers of sediment concentration and the sediment transport rate provided by the ACVP system. 
The ACVP provides reliable non-intrusive concentration estimates across the entire dilute suspension layer with shapes of the mean profiles following the expected Rouse profile [5], [11]. This confirms the expected validity and applicability of acoustic scattering based technologies in dilute sediment suspension flows. Inside the sheet flow layer, very good agreements with the CCP concentration measurements are found for $\phi \lesssim 0.35$ which covers $75 \%$ and $60 \%$ of the range of sheet flow layer thicknesses, for the S3 and S1 conditions, respectively. In the lower sheet flow layer associated with the sediment pick-up layer, we observe systematic over-estimations of the acoustic concentration measurements compared to the CCP concentration measurements. However, when normalized by the local mean concentrations (given by the $\mathrm{CCP}$ measurements as reference values), these relative differences are below $10 \%$ in the lowest and densest part of the sheet flow layer.

As expected from electric conductivity based measurement principle, the CCP technology provides accurate concentration profiles over the entire dense sheet flow layer associated with $\phi \gtrsim 0.08$. More unexpected is the ability to return accurate concentration estimations in the dilute suspension layer down to concentrations of the order of $1 \%$ for the $C C P_{1 \mathrm{~mm}}$ and $10 \%$ for $C C P_{2 m m}$, respectively. This demonstrates that with an appropriate vertical resolution (i.e., smaller than the sheet flow layer thickness), the CCP technology provides reliable instantaneous profile data outside its conventional range of application previously thought to be restricted to $\phi>5-8 \%$ for $C C P_{1 \mathrm{~mm}}$. This experiment marked the first deployment of the $C C P_{2 m m}$ instrument.

Acknowledgments: This research was funded by the Région Rhône-Alpes (COOPERA project and Explora Pro grant), the French national program EC2CO-LEFE MODSED, the European Community's Horizon 2020 Program through the Integrated Infrastructure Initiative HYDRALAB+ COMPLEX (no. 654110) and the French DGA funded ANR ASTRID Maturation project MESURE (no. ANR-16ASMA-0005-01). The authors are grateful for their support. The data used are available as supplementary material.

\section{References}

[1] J. A. Roelvink and I. Brøker, Coast. Eng., 21, 1-3, 163-191 (1993).

[2] D. Liu, X. Liu, X. Fu, and G. Wang, J. Geophys. Res. Earth Surf., 121, 4, 767-789, (2016).

[3] T. J. Hsu, J. T. Jenkins, and L. F. Liu, J. Geophys. Res., 108, C3, 14, (2003).

[4] J. T. Jenkins and D. M. Hanes, J. Fluid Mech., 370, 29-52, (1998).

[5] T. Revil-Baudard and J. Chauchat, J. Geophys. Res. Ocean., 118, 2, 619-634, (2013).

[6] B. M. Sumer, A. Kozakiewicz, J. Fredsøe, and R. Deigaard, J. Hydraul. Eng., 122, 10, 549-558, (1996).

[7] E. A. Cowen, R. D. Dudley, Q. Liao, E. A. Variano, and P. L.-F. Liu, Exp. Fluids, 49, 1, 77-88, (2010).

[8] H. Capart and L. Fraccarollo, Geophys. Res. Lett., 38, 20, L20402, (2011).

[9] D. Hurther, P. D. Thorne, M. Bricault, U. Lemmin, and J.-M. Barnoud, Coast. Eng., 58, 7, 594-605, (2011).

[10] T. Lanckriet, J. A. Puleo, and N. Waite, Ieee J. Ocean. Eng., 38, 1, 55-70, (2013).

[11] T. Revil-Baudard, J. Chauchat, D. Hurther, and P.-A. Barraud, J. Fluid Mech., 767, 1-30, (2015).

[12] G. E. Archie, Trans. AIME, 146, 1, 54-62, (1942).

[13] R. A. Bagnold, Phil. Trans. R. Soc. Lond., 249, 235-297, (1956).

[14] M. Bricault, Grenoble, INPG, (2006).

[15] T. O'Donoghue and S. Wright, Coast. Eng., 50, 3, 117-138, (2004). 